\title{
Analyse toxicologique avec mesure par « masse exacte». Caractérisation de métabolites urinaires par couplage UPLC/QTOF : apports et intérêts dans le cadre de la soumission chimique
}

\section{Toxicological analysis with exact mass measurement. Characterization of urinary metabolites by coupling UPLC/QTOF: contributions and interest in the context of chemical submission}

Luc Humbert ${ }^{\star}$, Camille Richeval

Laboratoire de Toxicologie et de Génopathies, Centre de Biologie Pathologie, Bd du Prof. J. Leclercq, 59037 Lille, France

\begin{abstract}
Résumé - Objectifs : Le métabolisme des médicaments conduit à des composés plus polaires facilitant leur élimination dans les urines. Peu de médicaments sont retrouvés inchangés dans les urines. Souvent pour mettre en évidence une soumission chimique, le toxicologue reçoit du sang et des urines prélevés plusieurs jours voire une semaine après les faits. Plus les faits seront éloignés des prélèvements, plus les chances de mettre en évidence la moindre trace d'une substance s'amenuisent. Le but de ce travail a été de mettre en place un protocole permettant d'obtenir des informations spectrales sur des métabolites de psychotropes excrétés en grande quantité dans les urines. Méthodes : Les urines de patients ayant ingéré massivement un médicament sont analysées par chromatographie liquide couplée à un spectromètre de masse hybride quadripôle-temps de vol (UPLC XEVO G2 QTOF). Le mode MS ${ }^{e}$ réalise une double acquisition simultanée : une à basse énergie d'accélération pour obtenir la masse exacte des composés, la seconde à haute énergie d'accélération pour fragmenter l'ensemble des composés. Les données sont traitées par des logiciels spécialisés dans la recherche et la caractérisation de métabolites (MetaboLynx ${ }^{\mathrm{TM}}$ ) et dans l'analyse des fragments de collision (MassFragment). Résultats : Dans les urines d'un patient ayant ingéré massivement du bromazépam, il a été possible de caractériser 3 métabolites majeurs. Lors d'une ingestion massive d'alimémazine, il a été possible de caractériser 5 métabolites majeurs. Conclusion : L'élargissement de la fenêtre de détection d'un composé dans des urines est possible par la caractérisation des métabolites.
\end{abstract}

\begin{abstract}
Mots clés : Soumission chimique, métabolites, masse exacte
Abstract - Objective: The metabolism of drugs leads to more polar compounds, facilitating urinary elimination. Few drugs are recovered unchanged in the urine. Often, to highlight a chemical submission, the toxicologist receives blood and urine for events dating back to several days to a week. If the time between the events and the biological sample is long, the chance of highlighting a product is small. The aim of this study was to establish a protocol to obtain spectral information on metabolites of psychoactive drugs excreted in large quantities in the urine. Methods: The urines of patients who ingested a massive amount of a drug are analyzed by liquid chromatography-hybrid quadrupole timeof-flight mass spectrometry (UPLC QTOF XEVO G2). The MS ${ }^{\mathrm{e}}$ mode performs a double simultaneous acquisition: a low-energy acceleration to obtain the exact mass of compounds, the second at high acceleration energy to fragment all of the compounds. The data are processed by specialized software for research and characterization of metabolites $\left(\right.$ MetaboLynx ${ }^{\mathrm{TM}}$ ), and analysis of collision fragments (MassFragment). Results: In the urine of a patient who ingested a massive amount of bromazepam, it was possible to characterize three major metabolites. Also, after a massive ingestion of alimemazine it was possible to characterize five major metabolites. Conclusion: Enlargement of the detection window of a compound in urine is possible by the characterization of its metabolites excreted in large amounts.
\end{abstract}

Key words: Chemical submission, metabolites, exact mass

Reçu le 15 mars 2012, accepté après modifications le 24 mai 2012

Publication en ligne le 22 juin 2012

\footnotetext{
^Correspondance : Luc Humbert, 1-humbert@chru-lille.fr
} 


\section{Introduction}

La soumission chimique ou médicamenteuse se définit comme l'administration à des fins criminelles (viol, actes de pédophilie) ou délictuelles (violences volontaires, vol) de substances psycho-actives à l'insu de la victime ou sous la menace. Si la plupart des observations concernent des jeunes femmes, à qui l'agresseur administre une substance afin de diminuer la résistance à l'acte sexuel, des enfants et des personnes âgées [1], tous les individus sont aussi des victimes potentielles.

Ce modus operandi n'est pas nouveau mais il a été, et est toujours, difficile à prouver en raison de nombreuses difficultés pré-analytiques et analytiques. Cependant, grâce à l'évolution constante des technologies, des méthodes de plus en plus sensibles et spécifiques par chromatographie liquide (CL) ou gazeuse $(\mathrm{CG})$ couplée à la spectrométrie de masse ou de masse tandem CL(CG)-SM(/SM) permettent de détecter maintenant lors d'un screening réalisé en mode «full scan» la plupart des molécules rencontrées [2]. Désormais, par ces méthodes, la prise unique d'un composé est souvent détectable quelques jours après administration dans le sang et plus d'une semaine dans les urines [3].

Les produits utilisables, ou utilisés, sont nombreux : médicaments, substances licites ou illicites. Certains sont en vente libre (éthanol) ou non réglementée (cas de certaines substances « naturelles », telles l'atropine ou la scopolamine) [4]. L'accès large et facile à Internet, moyen moderne, puissant, simple et discret permet d'acquérir à l'étranger de nombreuses substances mais aussi des techniques de préparations ou d'utilisation de celles-ci [5].

Pour l'agresseur, un produit idéal doit : avoir une demi-vie courte, être soluble dans les boissons, agir sur la mémoire de la victime (amnésie antérograde partielle ou totale des faits). La victime aura du mal à se rappeler précisément des faits et tardera à porter plainte, retardant ainsi la collecte des prélèvements biologiques hypothéquant fortement la détection du produit.

Pour le toxicologue, la caractérisation de la substance par l'analyse des prélèvements biologiques de la victime est extrêmement difficile compte tenu des propriétés physicochimiques et cinétiques (faible quantité, demi-vie courte). La qualité et la précocité des prélèvements biologiques sont déterminantes pour les analyses. L'idéal étant de disposer à la fois de prélèvements sanguins, urinaires et capillaires [4]. Il est aussi fondamental de connaître l'intervalle qui existe entre l'heure supposée des faits et le moment des prélèvements. Cet intervalle, qui pourra être de plusieurs jours, orientera le choix des matrices à analyser et permettra au toxicologue une bien meilleure interprétation des résultats. La connaissance d'un traitement médicamenteux ou d'une consommation plus ou moins habituelle de certaines substances par la victime avant et/ou après les faits est essentielle.

Plusieurs auteurs ont publié des études de prévalence de substances retrouvées dans les cas de soumission chimique [6-10]. Il en ressort qu'après l'éthanol, le cannabis, les benzodiazépines et hypnotiques sont fréquemment rencontrés.

La Société Française de Toxicologie Analytique (SFTA) a fait paraître fin 2002 un numéro spécial concernant la soumission chimique. Kintz aborde, dans l'éditorial, la nécessité d'utiliser des techniques séparatives couplées à la spectrométrie de masse et si possible de masse tandem [11].

Pour une prise en charge optimale de ces recherches, plusieurs organismes ou sociétés ont publié des recommandations. En France en 2003, l'AFSSAPS recommandait une liste de molécules à rechercher préférentiellement. La même année, la Society of Forensic Toxicologists (SOFT) recommandait une liste de molécules (incluant parfois un métabolite) à rechercher dans les urines avec des préconisations de limites de détection maximales. De nombreux auteurs ont aussi publié un protocole pour réaliser des criblages d'un grand nombre de composés avec une très grande sensibilité [12-17].

La fenêtre de détection d'un xénobiotique est une notion très importante, il est souvent demandé au toxicologue la durée de détection d'un produit après son administration. Il est très difficile de répondre car la fenêtre de détection dépend de nombreux facteurs, et relativement peu d'études ont été publiées sur le sujet [18]. Le problème du délai de détection des produits dans les prélèvements biologiques en cas de soumission chimique a fait l'objet de différents travaux. En effet, la SFTA en 2002 a mené une étude sur des volontaires ayant ingéré une benzodiazépine ou apparentée. L'étude a montré que les couplages CL-SM/SM étaient les plus sensibles et les mieux adaptés pour élargir la fenêtre de détection [3]. Verstraete a étudié également les fenêtres de détection des xénobiotiques dans le sang, les urines, la salive et les cheveux [19]. Ces derniers sont bien sûr la matrice de choix pour élargir la fenêtre de détection mais elle oblige néanmoins de revoir la victime 3 à 5 semaines après les faits pour le prélèvement, pénalisant le travail d'investigation du requérant. Il rappelle que les fenêtres de détection sont fonction de la dose, de la voie d'administration, de l'usage ponctuel ou répété, du choix du prélèvement, du seuil de détection de la technique utilisée, de la nature de la molécule ou du métabolite recherché, du pH et de la concentration urinaire, et pour finir, des variations interindividuelles de métabolisation. ElSohly et Salamone ont montré également la distribution exponentielle décroissante des cas positifs en fonction du temps écoulé entre les faits et les prélèvements. Au-delà de 72 h, la probabilité d'une analyse positive par les méthodes classiques (CG-SM) est presque nulle [6].

L'élargissement de la fenêtre de détection d'un produit dans les urines passe par la caractérisation des métabolites, le plus souvent présents plus longtemps et en beaucoup plus grande quantité. Un grand nombre de ceux-ci sont de moins en moins disponibles auprès des laboratoires pharmaceutiques. Les sociétés commerciales spécialisées dans la vente de produits purs ne disposent pas de tous ces produits ou les vendent à des prix parfois prohibitifs.

Plusieurs études ont montré l'intérêt de les rechercher, c'est le cas par exemple du zolpidem après une prise unique dans les urines, il n'est plus détecté $36 \mathrm{~h}$ après par une détection par chromatographie liquide couplée à un spectromètre de masse (CL-SM) et 36 à 96 heures après détection par CL-SM/SM $[3,20]$. Or, les victimes se manifestant parfois plusieurs jours après les faits, la caractérisation est beaucoup plus limitée. La synthèse de deux métabolites du zolpidem [21] a permis de détecter la prise de $10 \mathrm{mg}$ de ce médicament pendant au moins $144 \mathrm{~h} \mathrm{[22].}$ 
<smiles>O=C1CN=C(c2ccccn2)c2cc(Br)ccc2N1</smiles>

Bromazepam<smiles>CNc1ccc(Br)cc1C(=O)c1ccccn1</smiles>

Produit de clivage<smiles>O=C1Nc2ccc(Br)cc2C(c2ccccn2)=NC1O</smiles>

Hydroxy-bromazepam<smiles>Nc1c(O)cc(Br)cc1C(=O)c1ccccn1</smiles><smiles>CC</smiles>

Conjugaison
Produit de clivage hydroxylé

Fig. 1. Schéma métabolique du bromazépam.

Depuis maintenant quelques années, les couplages de systèmes chromatographiques avec des analyseurs mesurant la masse exacte se développent (temps de vol, orbitrap). Ils ouvrent de nouveaux champs d'investigation au toxicologue analyste [23-28]. Contrairement aux systèmes triple quadripolaire dont la limite de détection la plus basse est obtenue en mode dit MRM (Multi Reaction Monitoring), mode le plus sensible actuellement mais ne permettant de trouver que ce que l'on recherche et relativisant aussi grandement l'exhaustivité d'un criblage, l'acquisition en masse exacte a l'énorme avantage de se faire en mode dit « full scan » et non en mode ciblé. Cette acquisition présente l'avantage d'être plus sensible que le screening général effectué en mode « full scan» [2].

Des logiciels très puissants sont associés à ces technologies. Ils permettent des fonctionnalités et des recherches jusqu'alors impossibles ou incomplètes. Ils rendent possible de suspecter la présence ou non d'un produit en recherchant la présence de la masse exacte dans une acquisition en mode « full scan », sans avoir une solution standard. Bien évidemment, d'autres critères devront être pris en compte avant d'en affirmer la présence mais la modélisation in silico est désormais possible. Ils offrent aussi la possibilité après avoir sélectionné les voies de métabolisme connues d'un composé de rechercher et caractériser ses métabolites dans un échantillon urinaire.

Ce travail préliminaire a pour but d'enrichir une base de données de référence existante par la caractérisation des métabolites principaux des substances impliquées dans la soumission chimique en montrant la faisabilité de la méthode à partir d'échantillons urinaires de personnes admises dans les services de réanimation après une tentative d'autolyse médicamenteuse. Nous nous attacherons à détailler les outils et les performances d'un analyseur à temps de vol (TOF) tant sur le plan analytique qu'au niveau des traitements des données acquises par les logiciels.

\section{Matériels et méthode}

\section{1 Échantillons}

Les urines proviennent de patients admis dans un service d'urgences après une tentative d'autolyse médicamenteuse et pour lesquelles le laboratoire de toxicologie a été sollicité pour un screening toxicologique large en CL-SM/SM. Les urines pour lesquelles un xénobiotique est détecté seront conservées. Un flacon de $30 \mathrm{~mL}$ d'urine est maintenu à $-20{ }^{\circ} \mathrm{C}$ jusqu' aux analyses complémentaires. Parmi ces échantillons, le cas $\mathrm{n}^{\circ} 1$ correspond à une intoxication volontaire au bromazépam, dont le métabolisme est décrit (figure 1 ). Le cas $n^{\circ} 2$ correspond à une intoxication volontaire à l'alimémazine (figure 2), dont les voies métaboliques passent essentiellement par des sulfoxydations, des hydrolylations et des $\mathrm{N}$ desméthylations [29,30].

\subsection{Réactifs}

Tous les solvants utilisés sont de qualité HPLC grade. L'acide acétique, le borate de sodium, l'acide formique, le formate d'ammonium et la $\beta$-glucuronidase/arylsulfatase 


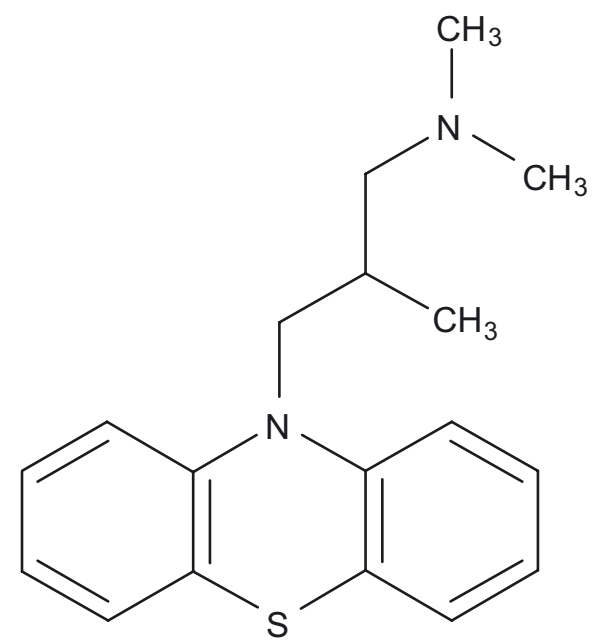

Fig. 2. Formule développée de l'alimémazine.

Helix pomatia proviennent de chez Sigma-Aldrich (SaintQuentin-Fallavier, France), le dichlorométhane de Carlo Erba (France), le diéthyléther de chez Panreac (France), l'acétonitrile et l'eau désionisée de chez Biosolve (France).

\subsection{Processus d'extraction}

Une déglucuronoconjuguaison et une désulfatation des métabolites conjugués sont réalisées sur deux aliquots de $500 \mu \mathrm{L}$ d'urine par $20 \mu \mathrm{L}$ de $\beta$ glucuronidase/arylsulfatase à $37{ }^{\circ} \mathrm{C}$ pendant 4 heures. Les échantillons sont extraits par un mélange de solvants organiques, composé de Dichlorométhane/Ether/Hexane/Isoamylalcool (30/50/20/0,5, v/v) (tableau I). Après agitation au Vortex pendant 3 minutes, les mélanges sont centrifugés pendant 5 minutes à 4000 tours/min. Les phases organiques sont récupérées puis séchées sous azote. Les extraits obtenus sont repris par $100 \mu \mathrm{L}$ de phase mobile.

\subsection{Conditions opératoires}

La séparation chromatographique est faite en chromatographie liquide Ultrahaute performance UPLC (Waters) équipé d'une colonne ACQUITY HSS C $18150 \mathrm{~mm} \times 2 \mathrm{~mm}, 1,8 \mu \mathrm{m}$ maintenue à $50{ }^{\circ} \mathrm{C}$ dans un four thermostaté. L'élution de la colonne est faite par un gradient acétonitrile $/ 0,1 \%$ acide formique - tampon formate d' ammonium pH 3,0 (tableau II).

L'acquisition est faite par un spectromètre de masse hybride quadripôle - temps de vol (QTOF) G2 QTOF (Waters) utilisé en mode $\mathrm{MS}^{\mathrm{e}}$. Les principaux réglages sont regroupés dans le tableau III.

\subsection{Traitement des données}

L'analyse des données SM/SM est contrôlée avec MetaboLynx ${ }^{\mathrm{TM}}$. Ce logiciel répertorie les voies métaboliques connues permettant ainsi, à partir de la formule brute d'une molécule mère, le calcul de la masse exacte des métabolites
Tableau I. Protocole d'extraction des urines.

\begin{tabular}{l|c|c}
\hline & $\begin{array}{c}\text { Extraction } \\
\text { en milieu } \\
\text { acide }\end{array}$ & $\begin{array}{c}\text { Extraction } \\
\text { en milieu } \\
\text { alcalin }\end{array}$ \\
\hline Urine déglucuronoconjuguée & $0,5 \mathrm{~mL}$ & $0,5 \mathrm{~mL}$ \\
Méthylclonazépam (étalon interne) & $0,1 \mathrm{~mL}$ & $0,1 \mathrm{~mL}$ \\
Acide acétique 1\% & $0,2 \mathrm{~mL}$ & $/$ \\
Tampon borate saturé pH 9,0 & $/$ & $0,2 \mathrm{~mL}$ \\
Mélange d'extraction* & $3 \mathrm{~mL}$ & $3 \mathrm{~mL}$ \\
\hline
\end{tabular}

* Mélange d'extraction : Dichlorométhane/Ether/Hexane/Isoamylalcool $(30 / 50 / 20 / 0,5, \mathrm{v} / \mathrm{v})$.

Tableau II. Conditions du gradient UPLC.

\begin{tabular}{c|c|c}
\hline Temps (min) & $\begin{array}{c}\text { A : Tampon formate } \\
(\%)\end{array}$ & $\begin{array}{c}\text { B : Acétonitrile } \\
(\%)\end{array}$ \\
\hline 0 & 87 & 13 \\
0,5 & 87 & 13 \\
10 & 50 & 50 \\
10,75 & 5 & 95 \\
12,25 & 5 & 95 \\
12,5 & 87 & 13 \\
15 & 87 & 13 \\
\hline
\end{tabular}

connus puis de les extraire au sein d'un chromatogramme. MetaboLynx $^{\mathrm{TM}}$ présente les masses calculées (théoriques), mesurées, les temps de rétention, les surfaces des pics, les spectres de masses pour chacun des métabolites recherchés (figure 3 ).

Le spectre moyenné de chaque pic de métabolite suspecté acquis à haute énergie de collision (rampe de 10 à $40 \mathrm{eV}$ ) est transféré dans MassFragment. Une analyse comparative est faite sur les 20 fragments les plus abondants de chaque spectre de masse. Celle-ci consiste à vérifier, avec les règles de fragmentations connues en spectrométrie de masse, la cohérence des fragments obtenus avec la formule développée de chaque métabolite recherché. En fin d'analyse, pour chaque fragment le logiciel propose une «formule développée» pour le fragment en lien avec la formule développée de ce métabolite, dessiné via Chemsketch. Une masse exacte est calculée, un écart de masse inférieur à 3 ppm par rapport à la masse mesurée est considéré comme acceptable. Les résultats sont regroupés dans un tableau donnant pour chacune des 20 masses exactes la pertinence d'appartenir à un fragment spécifique de la molécule (figure 4).

\subsection{Nomenclature}

Les métabolites caractérisés sont inclus dans la base de données spectrales de la manière suivante :

- composé-m(OH) quand la connaissance du métabolisme montre que le composé parent s'hydroxyle; 


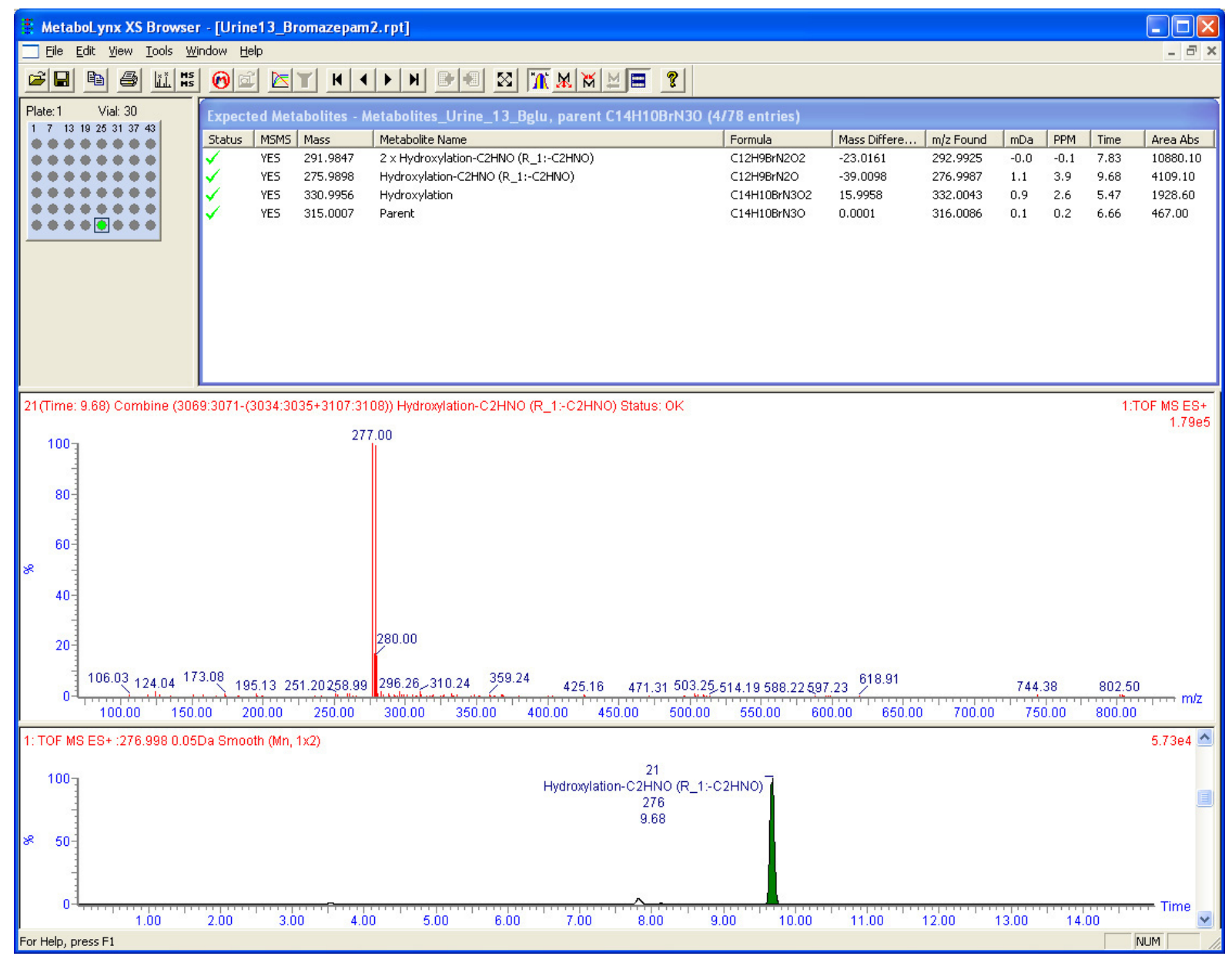

Fig. 3. (Couleur dans la version en ligne) Rapport d'analyse de MetaboLynx ${ }^{\mathrm{TM}}$.

Tableau III. Paramètres de source et d'acquisition du XEVO G2 QTOF.

\begin{tabular}{l|l}
\hline Paramètres de source & Paramètres d'acquisition \\
\hline Ionisation : Électrospray positif & Mode d'acquisition : MS \\
Tension du capillaire $: 1 \mathrm{kV}$ & Tension de cône $: 20 \mathrm{~V}$ \\
Température de la source $: 140{ }^{\circ} \mathrm{C}$ & $\underline{\text { Fonction } 1}:$ \\
Débit de cône gaz $: 20 \mathrm{~L} / \mathrm{h}$ & - Gamme de masse $100-1000 \mathrm{~m} / \mathrm{z}$ \\
Température de désolvation $: 450{ }^{\circ} \mathrm{C}$ & $\underline{\text { Fonction } 2}:$ \\
Débit de gaz de désolvatation $: 1000 \mathrm{~L} / \mathrm{h}$ & - Gamme de masse $50-1000 \mathrm{~m} / \mathrm{z}$ \\
& - Rampe de collision $: 10-40 \mathrm{eV}$ \\
\hline
\end{tabular}

- composé-m(O) quand la connaissance du métabolisme fait apparaître l'ajout d'un oxygène $(\mathrm{O})$ (par exemple soit hydroxylation soit sulfoxylation);

- composé-m(-CH3) quand la connaissance du métabolisme montre que le composé parent peut être déméthylé.

\section{Résultats}

\section{Cas $n^{\circ} 1$}

Il s'agit d'une femme de 34 ans amenée par le SAMU en réanimation après avoir ingéré suite à une tentative d'autolyse 1 boîte de LEXOMIL ${ }^{\circledR}(30$ comprimés de $6 \mathrm{mg})$. Le screening toxicologique réalisé dans les urines en CL-SM/SM a confirmé la présence de bromazépam.

La connaissance du métabolisme de cette molécule (figure 1) permet de paramétrer le logiciel MetaboLynx ${ }^{\mathrm{TM}}$ pour le calcul des masses exactes correspondant au 3hydroxybromazépam, au 2-(2-amino-5-bromo-benzoyl) pyridine [produit de clivage] et au 2-(2-amino-5-bromo-3hydroxybenzoyl) pyridine [produit de clivage hydroxylé] qui sont ensuite recherchées dans les différents chromatogrammes acquis en mode $\mathrm{MS}^{\mathrm{e}}$.

Trois pics intenses possédant chacun l'une des trois masses exactes pouvant correspondre au 3-hydroxybromazépam, au produit de clivage et au produit de clivage hydroxylé sont 


\section{Results}

Processing is complete. Results are presented below:

Input:

\begin{tabular}{|l|l|l|}
\hline ID (job) & 73 \\
\hline Mass (Da) & 291.9847 \\
\hline & Formula & C $_{12} \mathrm{H}_{9} \mathrm{~N}_{2} \mathrm{O}_{2} \mathrm{Br}$ \\
\hline
\end{tabular}

Experiment:

\begin{tabular}{|c|c|}
\hline Product ion(s) (Da) & $\begin{array}{l}106.0293107 .0323124 .0398167 .0612168 .0684169 .0754196 .0635213 .0661 \\
214.0733274 .9820275 .9849276 .9798277 .9831292 .9924293 .9959294 .9904 \\
295.993778 .034479 .037596 .0449 \\
+/-0.01 \text { in positive mode, structure filter off }\end{array}$ \\
\hline DBE & -10 to 50 \\
\hline Electron count & both \\
\hline Maximum $\mathrm{H}$ deficit & 6 \\
\hline Fragment number of bonds & 4 \\
\hline Scoring & $\begin{array}{l}\text { aromatic: } 6 \text {, multiple: } 4 \text {, ring: } 2 \text {, phenyl: } 8 \text {, other: } 1 \\
\text { H-deficit: } 0 \text {, hetero modifier: } 0.5 \text {, alpha penalty: } 5 \text {, max score: } 16\end{array}$ \\
\hline Order: & mass \\
\hline
\end{tabular}

Results:

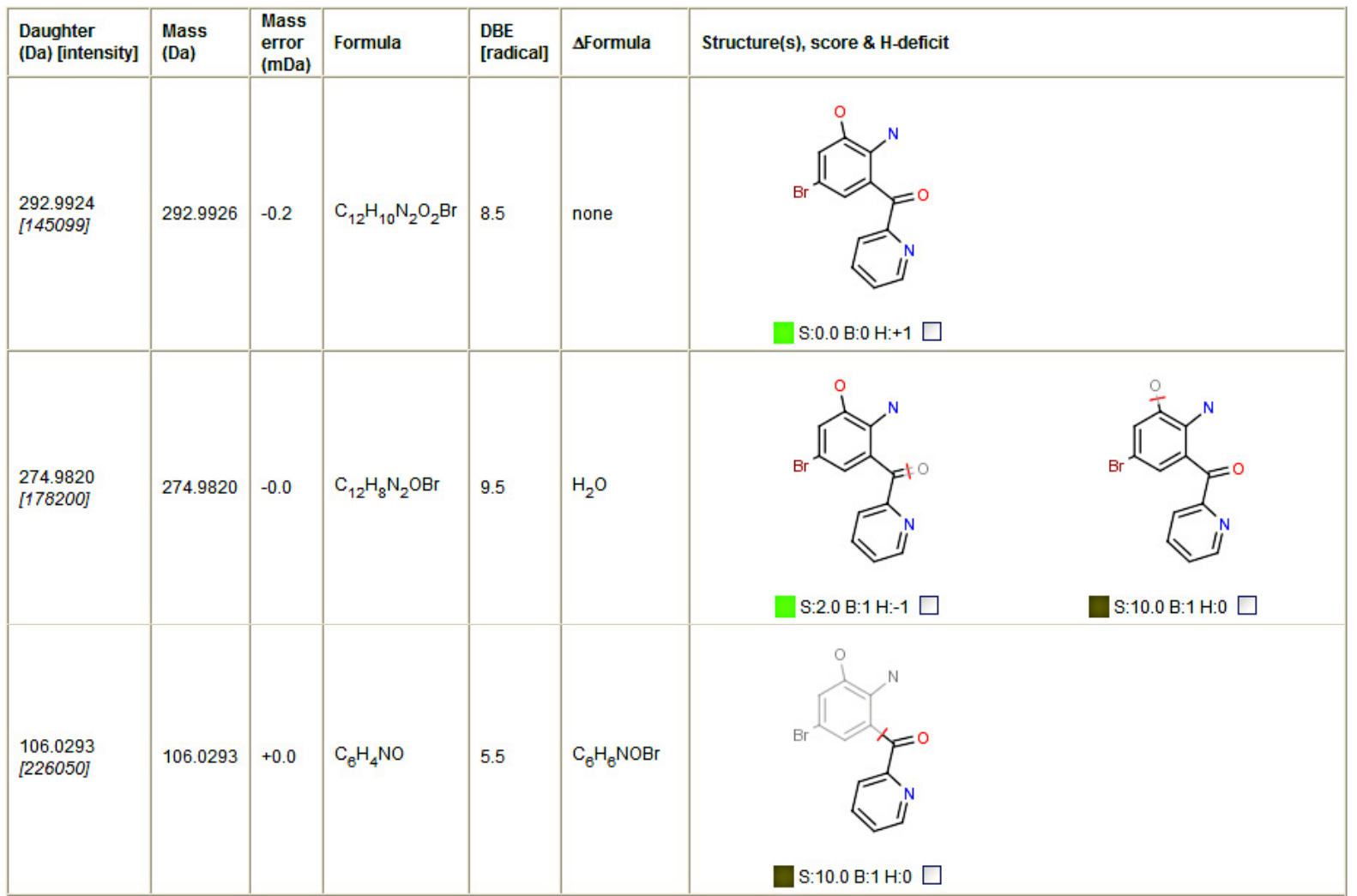

Fig. 4. (Couleur dans la version en ligne) Rapport d'analyse des fragments du 2-(2-amino-5-bromo-3-hydroxybenzoyl) pyridine obtenus à haute énergie de collision. 


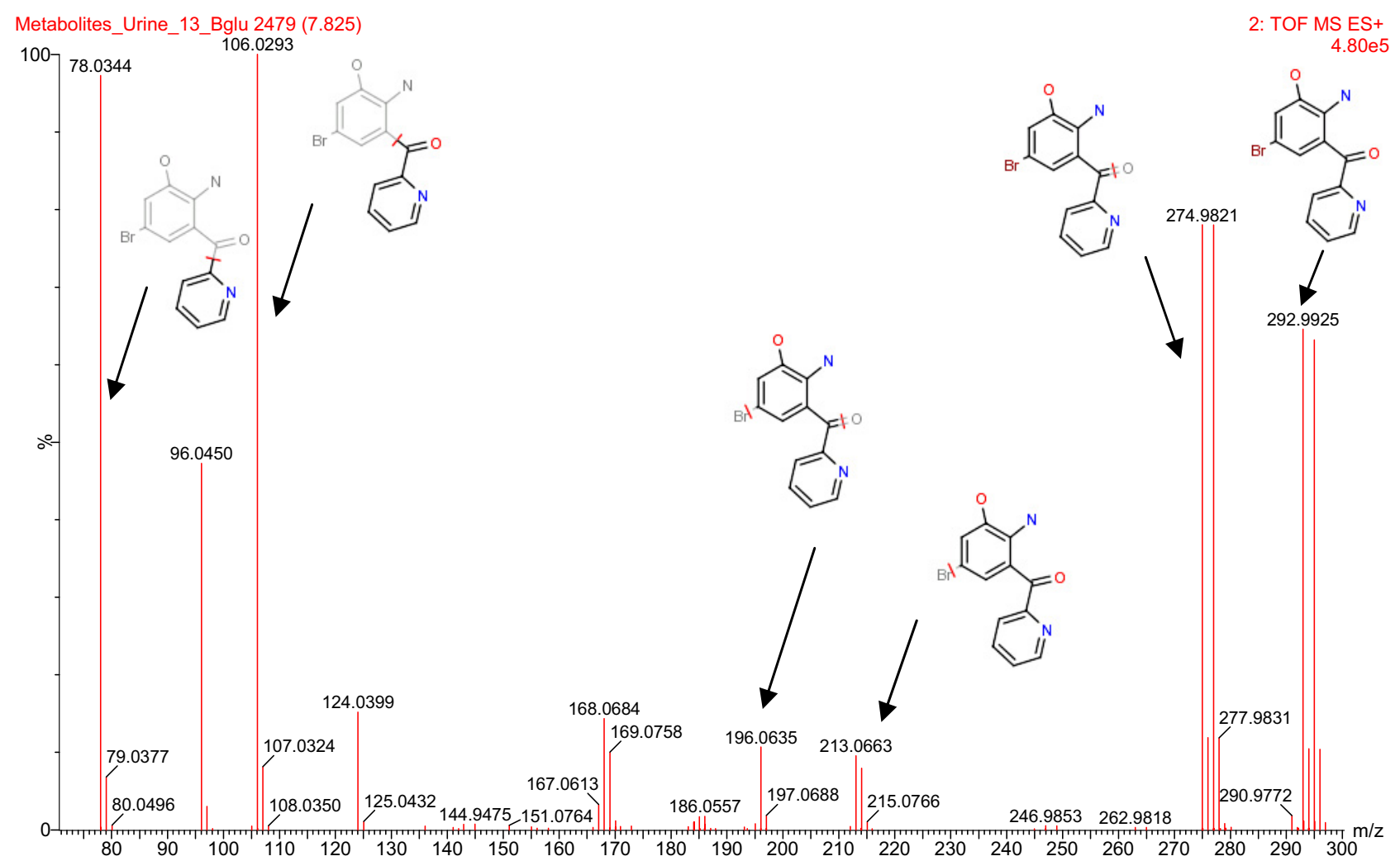

Fig. 5. (Couleur dans la version en ligne) Résultat de MassFragment pour le métabolite potentiel correspondant au 2-(2-amino-5-bromo-3hydroxybenzoyl) pyridine au temps de rétention $7,8 \mathrm{~min}$.

détectés. L'analyse par MassFragment de leurs spectres respectifs acquis à haute énergie de collision avec leurs formules développées ont permis de confirmer la cohérence des fragments obtenus (figure 5). Trois nouvelles entrées sont alors ajoutées à la librairie développée au laboratoire, elles contiennent chacune la formule brute du métabolite, le temps de rétention ainsi que les masses exactes des fragments les plus abondants validés par MassFragment (tableau IV).

\section{Cas $n^{\circ} 2$}

Il s'agit d'une femme de 55 ans amenée par le SAMU en réanimation après avoir ingéré un flacon de THERALENE ${ }^{\circledR}$ suite à une tentative d'autolyse. Le screening toxicologique réalisé en CL-SM/SM a mis en évidence la présence de l'alimémazine.

Les masses exactes calculées de métabolites correspondant à une hydroxylation, une sulfoxylation et/ou une désalkylation sont recherchées par MetaboLynx ${ }^{\mathrm{TM}}$ dans les différents chromatogrammes acquis en mode $\mathrm{MS}^{\mathrm{e}}$.

Plusieurs pics importants sont détectés correspondant potentiellement à des métabolites issus des voies de métabolisation de l'alimémazine. En procédant de la même manière, cinq nouvelles entrées sont ajoutées à la base de données, elles contiennent chacune la formule brute du métabolite, le temps de rétention ainsi que les masses exactes des fragments les plus abondants validés par MassFragment (tableau V).

\section{Discussion}

Les xénobiotiques, souvent liposolubles, sont transformés dans l'organisme par des enzymes qui permettent de les rendre hydrosolubles donc plus facilement excrétables dans les urines. Parmi ces enzymes, des réactions effectuées par des monooxygénases à cytochrome $\mathrm{P} 450$ permettent des réactions d'hydroxylation, de désalkylations... [31]. L'excrétion urinaire d'un médicament et de ses métabolites correspond souvent à une fraction de la dose administrée. Seulement $2 \%$ de la dose administrée de bromazépam se retrouve dans les urines sous forme inchangée, alors que le dérivé glucuronoconjugué du 3hydroxybromazépam correspond à environ $27 \%$ de la dose et que la somme des dérivés conjugués du produit de clivage et du produit de clivage hydroxylé correspondent environ à $40 \%$ de la dose administrée (figure 6) [27,28].

La connaissance des voies métaboliques des xénobiotiques médicamenteux, conjugués à l'utilisation de plusieurs logiciels puissants ont permis de rechercher et de caractériser la présence de métabolites spécifiques dans des urines sans en avoir nécessairement les principes actifs. Cette étude s'est portée sur la recherche de métabolites de phase I puisque les urines sont préalablement traitées par $\beta$ glucuronidase et sulfatase. Seuls les métabolites présents dans les urines en grande quantité ont retenu notre intérêt, car ce sont eux qui permettront d'élargir la fenêtre de détection d'un composé dans les urines.

L'association de MetaboLynx ${ }^{\mathrm{TM}}$ et MassFragment ont permis d'identifier 3 métabolites du bromazépam et 5 de l'alimémazine. Cependant, la présence dans un chromatogramme 
Tableau IV. Paramètres d'identification des métabolites du bromazépam.

\begin{tabular}{l|c|c|l|l|l}
\hline Nom & Formule brute & Temps de rétention & Fragment 1 & Fragment 2 & Fragment 3 \\
\hline Bromazépam-m $(\mathrm{OH})$ & C14H10BrN3O2 & 6,03 & $\mathrm{f}: 286,9820$ & $\mathrm{f}: 273,9980$ & \\
Bromazépam-m (Produit de clivage hydroxylé) & $\mathrm{C} 12 \mathrm{H} 9 \mathrm{~N} 2 \mathrm{O} 2 \mathrm{Br}$ & 7,82 & $\mathrm{f}: 274,9820$ & $\mathrm{f}: 78,0344$ & $\mathrm{f}: 106,0293$ \\
Bromazépam-m (Produit de clivage) & $\mathrm{C} 12 \mathrm{H} 9 \mathrm{~N} 2 \mathrm{OBr}$ & 10,07 & $\mathrm{f}: 106,0293$ & $\mathrm{f}: 78,0344$ & $\mathrm{f}: 258,9871$ \\
\hline
\end{tabular}

Tableau V. Paramètres d'identification des métabolites de l'alimémazine.

\begin{tabular}{l|l|c|l|l|l|l}
\hline Nom & Formule brute & Temps de rétention & Fragment 1 & Fragment 2 & Fragment 3 & Fragment 4 \\
\hline Alimémazine-m (+O) & C18H22N2OS & 6,3 & $\mathrm{f}: 100,1126$ & $\mathrm{f}: 228,0483$ & $\mathrm{f}: 196,0762$ & $\mathrm{f}: 58,0657$ \\
Alimémazine-m (+O) & C18H22N2OS & 5,02 & $\mathrm{f}: 100,1126$ & $\mathrm{f}: 198,0377$ & $\mathrm{f}: 267,1497$ & $\mathrm{f}: 58,0657$ \\
Alimémazine-m (+O-CH3) & C17H20N2OS & 4,9 & $\mathrm{f}: 199,0456$ & $\mathrm{f}: 86,0970$ & $\mathrm{f}: 198,0377$ & $\mathrm{f}: 212,0534$ \\
Alimémazine-m (+O-CH3) & C17H20N2OS & 6,17 & $\mathrm{f}: 86,0970$ & $\mathrm{f}: 228,0483$ & $\mathrm{f}: 196,0762$ & $\mathrm{f}: 215,0405$ \\
Alimémazine-m (-CH3) & C17H20N2S & 8,08 & $\mathrm{f}: 86,0970$ & $\mathrm{f}: 212,0534$ & $\mathrm{f}: 180,0813$ & $\mathrm{f}: 254,1003$ \\
\hline
\end{tabular}

Metabolites Urine_13 Bglu

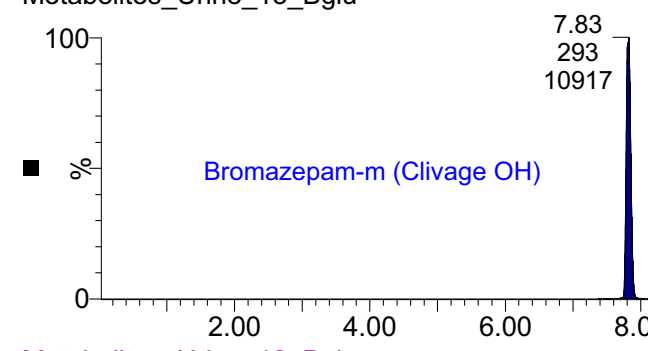

Metabolites_Urine_13_Bglu

100

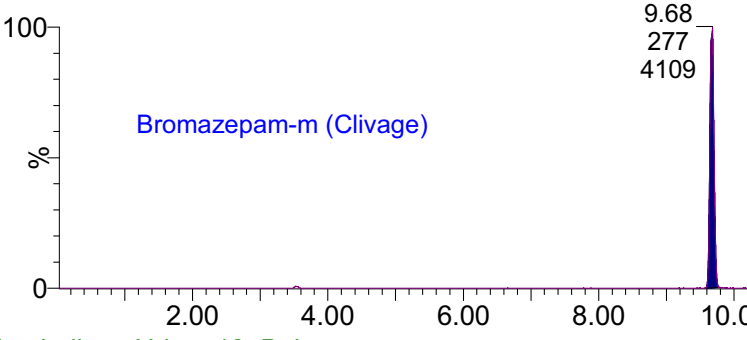

Metabolites_Urine_13_Bglu

100
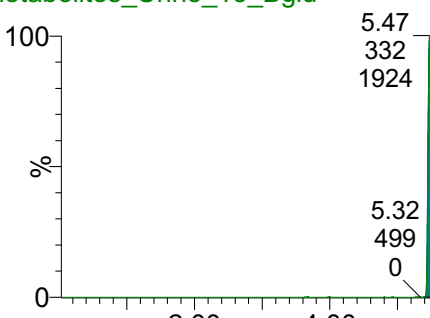

\section{7}

24

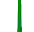

Bromazepam-m $(\mathrm{OH})$

32

499

2.004 .00

Metabolites_Urine_13_Bglu

$\begin{array}{llllll}6.00 & 8.00 & 10.00 & 12.00 & 14.00\end{array}$

1: TOF MS ES+

316.009 0.0100Da

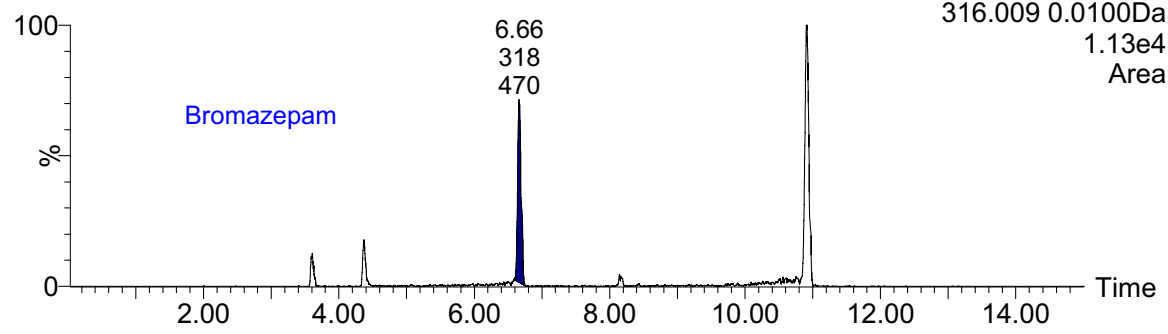

Fig. 6. (Couleur dans la version en ligne) Identification du bromazépam et de ses métabolites dans les urines d'un patient ayant ingéré du bromazépam. 
d'un pic ayant la masse exacte d'un métabolite recherché ne suffit pas pour le caractériser. Pour une même masse exacte, il existe de nombreux isomères.

Ainsi lorsqu'un médicament est hydroxylé ou sulfoxylé, sa masse exacte se trouve augmentée de $\mathrm{M}+$ 15,9949. C'est le cas du bromazépam qui est métabolisé en 3-hydroxybromazépam, c'est également le cas de deux métabolites de l'alimémazine, molécule pour laquelle 2 pics ont été repérés avec une masse exacte incrémentée de $\mathrm{M}+$ 15,9949 par rapport à la molécule mère, sans que la structure exacte puisse être définie (hydroxylation ou sulfoxydation).

Le métabolisme du bromazépam conduit aussi à la formation du 2-(2-amino-5-bromo-benzoyl) pyridine [produit de clivage] et au 2-(2-amino-5-bromo-3-hydroxybenzoyl) pyridine [produit de clivage hydroxylé], dont les masses exactes sont M - 39,0098 pour le produit de clivage $\mathbf{M}-23,0161$ pour le produit de clivage hydroxylé.

De même, à côté des réactions d'hydroxylation et de sulfoxydation, l'alimémazine peut subir une réaction de désalkylation (perte d'un groupement méthyle) qui conduira à un métabolite ayant une masse exacte de $\mathrm{M}-14,0157$, ce composé désalkylé pouvant être lui-même hydroxylé ou sulfoxylé avec comme masse exacte $\mathrm{M}+1,9791$ par rapport à l'alimémazine. Ainsi, trois pics supplémentaires importants ont été repérés correspondant pour l'un à une perte d'un groupement méthyle et pour les deux autres à la perte d'un groupement méthyle, plus l'ajout d'un oxygène dans la formule de l'alimémazine [32].

L'enrichissement de base de données de référence, par l'ajout de paramètres caractérisant quelques métabolites principaux de xénobiotiques a plusieurs intérêts majeurs [33]. Le premier, dans le cas d'une identification d'un principe actif à l'état de traces dans une matrice biologique, la possibilité de détecter simultanément un ou plusieurs métabolites permettra de confirmer sa présence, l'autre est de permettre un élargissement important de la fenêtre de détection d'un composé, cet aspect est déterminant dans le cadre de la caractérisation d'une soumission chimique où le toxicologue reçoit en première intention du sang et de l'urine.

\section{Conclusion}

Pour identifier des composés inconnus sans aucun a priori par UPLC QTOF, il est nécessaire de s'appuyer pour un screening large sur une base de données renfermant les informations permettant de les caractériser. Cette identification pour les analyseurs de masse exacte repose sur la connaissance d'une formule brute (permettant de calculer sa masse exacte le plus souvent protonée), d'un temps de rétention dans des conditions chromatographiques définies et standardisées et idéalement les masses exactes d'un ou plusieurs de ses fragments spécifiques obtenus après des collisions. Ceci est relativement facile à collecter et à compiler quand on possède une solution pure de chacun des composés. Un grand nombre de composés sont à rechercher, il est de plus en plus difficile et/ou coûteux voire impossible (sauf à les faire synthétiser) d'obtenir quelques mg de tous ces métabolites. Une possibilité serait de les obtenir après incubation en utilisant des microsomes hépatiques humains $[34,35]$.
Grâce à ce procédé il est possible d'élargir la fenêtre de détection d'un composé par la caractérisation d'un ou plusieurs de ses métabolites urinaires présents en beaucoup plus grande quantité et qui persistent plus longtemps. Il permet aussi de conforter la présence d'un composé dans du sang mais surtout dans l'urine (ou la bile) en y associant la présence d'un ou plusieurs de ses métabolites.

Conflits d'intérêts. Les auteurs déclarent ne pas avoir de conflits d'intérêts.

\section{Références}

1. Kintz P, Cirimele V, Villain M, Tracqui A, Ludes B. Soumission chimique : approches pratiques en toxicologie médico-légale. Annal Toxicol Anal. 2002; 14: 361-364.

2. Humbert L, Grisel F, Richeval C, Lhermitte M. Screening of xenobiotics by ultra-performance liquid chromatography-mass spectrometry using in-source fragmentation at increasing cone voltages: library constitution and an evaluation of spectral stability. J Anal Toxicol. 2010; 34(9): 571-580.

3. Kintz P. Soumission chimique : prise en charge toxicologique. Ann Toxicol Anal. 2003; 15: 239-242.

4. Gaulier JM, Fonteau F, Jouanel E, Lachâtre G. Les substances de la soumission chimique : aspects pharmacologiques et analytiques. Annal Biol Clin. 2004; 62(5): 529-538.

5. Dumestre-Toulet V, Verstraete A. Hallucinogènes et Internet ou le cyberspace psychédélique. Toxicorama. 1998; 10: 98-105.

6. ElSohly MA, Salamone SJ. Prevalence of drugs used in cases of alleged sexual assault. J Anal Toxicol. 1999; 23: 141-146.

7. Hindmarch I, ElSohly M, Gambles J, Salamone S. Forensic urinalysis of drug in cases of alleged sexual assault. J Clin Forensic Med. 2001; 8: 197-205.

8. Questel F, Diament-Berger O, Lagier G. Soumission médicamenteuse : analyse d'une série de résultats toxicologiques urinaires. Ann Toxicol Anal. 2001; 13: 121-122.

9. Questel F, Lagier G, FoDjezzra D, Dally S, Elkaharrat D, Diamant-Berger O. Usage criminel de produits psychoactifs : analyse d'une série parisienne. Ann Toxicol Anal. 2002; 14: $371-380$

10. Enquête soumission chimique, Agence française de sécurité sanitaire des produits de santé. Document consulté sur le site http://afssaps.sante.fr/pdf/10/enqsch.pdf en juillet 2003.

11. Kintz P. Éditorial : la soumission chimique. Ann Toxicol Anal. 2002; 14(4): 359.

12. Negrusz A, Gaensslen RE. Analytical developments in toxicological investigation of drug-facilitated sexual assault. Anal Bioanal Chem. 2003; 376(8): 1192-1197.

13. Juhascik M, Le NL, Tomlinson K, Moore C, Gaensslen RE, Negrusz A. Development of an analytical approach to the specimens collected from victims of sexual assault. J Anal Toxicol. 2004; 28(6): 400-406.

14. Quintela O, Sauvage FL, Charvier F, Gaulier JM, Lachâtre G, Marquet P. Liquid chromatography-tandem mass spectrometry for detection of low concentrations of 21 benzodiazepines, metabolites, and analogs in urine: method with forensic applications. Clin Chem. 2006; 52(7): 1346-1355.

15. Pépin G. Aspects analytique, toxicologique, judiciaire de la soumission chimique : dix ans d'expérience. Ann Pharm Fr. 2010; 68(2): 61-75. 
16. Ishida T, Kudo K, Hayashida M, Ikeda N. Rapid and quantitative screening method for 43 benzodiazepines and their metabolites, zolpidem and zopiclone in human plasma by liquid chromatography/mass spectrometry with a small particle column. J Chromatogr B Analyt Technol Biomed Life Sci. 2009; 877(25): 2652-2657.

17. Dresen S, Ferreirós N, Gnann H, Zimmermann R, Weinmann W. Detection and identification of 700 drugs by multi-target screening with a 3200 Q TRAP LC-MS/MS system and library searching. Anal Bioanal Chem. 2010; 396(7): 2425-2434.

18. Vandevenne M, Vandenbussche H, Verstraete A. Detection time of drugs of abuse in urine. Acta Clin Belg. 2000; 55: 323-333.

19. Verstraete A. Fenêtre de détection des xénobiotiques dans le sang, les urines, la salive et les cheveux. Ann Toxicol Anal. 2002; 14(4): 390-394.

20. Villain M, Chèze M, Tracqui A, Ludes B, Kintz P. Windows of detection of zolpidem in urine and hair: application to two drug facilitated sexual assaults. Forensic Sci Int. 2004; 143: 157161.

21. Klupsch F, Houssin R, Humbert L, Imbenotte M, Henichart JP, Lhermitte M. Major metabolites of zolpidem: expeditious synthesis and mass spectra. Chem Pharm Bull (Tokyo). 2006; 54(9): 1318-1321.

22. Richeval C, Rifflet A, Humbert L, Imbenotte M, Houssin R, Lhermitte L. Élargissement de la fenêtre de détection du zolpidem par la recherche de ses métabolites urinaires dans le cadre de la soumission chimique. Ann Toxicol Anal. 2008; 20(2): 79-83.

23. Ojanpera S, Pelander A, Pelzing M, Krebs I, Vuori E, Ojanpera I. Isotopic pattern and accurate mass determination in urine drug screening by liquid chromatography/time of flight mass spectrometry. Rapid Commun Mass Spectrom. 2006; 20: 11611167.

24. ElSohly MA, Gul W, Murphy TP, Avula B, Khan IA. LC-(TOF) MS analysis of benzodiazepines in urine from alleged victims of drug-facilitated sexual assault. J Anal Toxicol. 2007; 31: 505-514.
25. Polettini A, Gottardo R, Pascali JP, Tagliaro F. Implementation and performance evaluation of a database of chemical formulas for the screening of pharmaco/toxicologically relevant compounds in biological samples using electrospray ionization-time-of flight mass spectrometry. Anal Chem. 2008; 80: 3050-3057.

26. Kaufmann A, Butcher P, Maden K, Widmer M. Ultraperformance liquid chromatography coupled to time of flight mass spectrometry (UPLC-TOF): a novel tool for multiresidue screening of veterinary drugs in urine. Anal Chim Acta. 2007; 586: 13-21.

27. Kolmonen M, Leinonen A, Pelander A, Ojanpera I. A general screening method for doping agents in human urine by solid phase extraction and liquid chromatography/time of flight mass spectrometry. Anal Chim Acta. 2007; 585: 94-102.

28. Baselt RC. Disposition of drugs and chemicals in Man, Sixth edition Biomedical Publication, 2002: 116-117.

29. Dictionnaire VIDAL 2011, $87^{\mathrm{e}}$ édition.

30. Hewick DS, Beckett AH. The metabolism in vitro of phenothiazine drugs. Biochem J. 1971; 122(5): 59-60.

31. Guengerich FP. Cytochrome P450s and other enzymes in drug metabolism and toxicity. AAPS J. 2006; 8(1): 101-111.

32. Sauvage FL, Gaulier JM, Lachâtre G, Marquet P. Pitfalls and prevention strategies for liquid chromatography-tandem mass spectrometry in the selected reaction-monitoring mode for drug analysis. Clin Chem. 2008; 54(9): 1519-1527.

33. Sauvage FL, Picard N, Saint-Marcoux F, Gaulier JM, Lachâtre $\mathrm{G}$, Marquet P. General unknown screening procedure for the characterization of human drug metabolites in forensic toxicology: applications and constraints. J Sep Sci. 2009; 32(18): 3074-3083.

34. Beaune P. Human cytochromes P450. Applications in pharmacology. Therapie. 1993; 48(6): 521-526.

35. Beaune P, Guengerich FP. Human drug metabolism in vitro. Pharmacol Ther. 1988; 37(2): 193-211. 